\title{
A case report of concurrence of aberrant extracranial internal carotid artery and nonrecurrent laryngeal nerve in a patient of advanced thyroid carcinoma invading hypopharynx
}

\author{
Feng Wen ${ }^{1}$, Ruiling Xie ${ }^{2}$, Hong Shen ${ }^{1}$, Enmin Zhao ${ }^{1}$ \\ ${ }^{1}$ Department of Otolaryngology, Head \& Neck Surgery, Peking University First Hospital, Beijing, China; ${ }^{2}$ Department of Nasopharyngeal \\ Carcinoma, Sun Yat-sen University Cancer Center, Guangzhou, China \\ Correspondence to: Enmin Zhao. Department of Otolaryngology, Head \& Neck Surgery, Peking University First Hospital, No. 8 Xishiku Street, \\ Western District, Beijing 100034, China. Email: enmin_ent@sina.com.
}

\begin{abstract}
Aberrant extracranial internal carotid artery and nonrecurrent laryngeal nerve (NRLN) are neurovascular anomalies, which clinicians should be aware of to prevent severe complications while performing transoral or open surgery in the head and neck area. These two variants can occur simultaneously because of similar etiology of embryological maldevelopment of arch arteries. In this study, a 64-yearold woman complaining of hoarseness presented a bulge in the left hypopharynx. A contrasted computed tomography scan indicated left thyroid mass with hypopharynx invasion, accompanied by right aberrant extracranial internal carotid artery and subclavian artery. Fine needle aspiration biopsy of the mass revealed papillary thyroid carcinoma (PTC). She underwent total thyroidectomy, partial hypopharyngectomy, neck dissection, and tracheotomy. Intraoperatively, a right NRLN was found above the level of the cricothyroid junction that entered the larynx. The patient did not present severe complications postoperatively. Radioactive iodine therapy was applied after the surgery. At the 3-year follow-up, she remained cancer-free, and the hoarseness has improved. This is the first report of concurrent aberrant extracranial internal carotid artery and NRLN. NRLN of right side can be predicted in the computed tomography scan by the sign of the right subclavian artery origining from the left side of the aortic arch and running an abnormal course between the esophagus and the vertebra. Meanwhile, aberrant extracranial internal carotid artery may occur simultaneously. Recognition of aberrant neurovascular structures enables clinicians to prevent severe surgical complications.
\end{abstract}

Keywords: Aberrant extracranial internal carotid artery (aberrant extracranial ICA); nonrecurrent laryngeal nerve (NRLN); thyroidectomy; head and neck surgery; case report

Submitted Aug 14, 2020. Accepted for publication Feb 04, 2021.

doi: 10.21037/gs-20-663

View this article at: http://dx.doi.org/10.21037/gs-20-663

\section{Introduction}

Aberrant extracranial internal carotid artery (ICA) is a vascular anomaly. The physicians should be aware of this anomaly because of the high risk of injury during endoscopy or open surgical procedures (1). The nonrecurrent laryngeal nerve (NRLN) is a rare anatomical variation with an overall pooled prevalence of only $0.7 \%$ (2). However, NRLN is associated with a high risk of nerve injury. It directly enters the larynx arising from the vagus nerve in the neck, instead of descending into mediastinum as the RLN (3). About $86.7 \%$ of the right NRLN cases accompanied by the aberrant right subclavian artery facilitates the recognition of NRLN (2). The two anomalies mainly result from the embryological maldevelopment of arch arteries $(1,2)$, facilitating their simultaneous occurrence. Herein, we reported a patient with advanced thyroid carcinoma invading hypopharynx but rarely harbored NRLN and aberrant extracranial ICA and emphasized the importance 


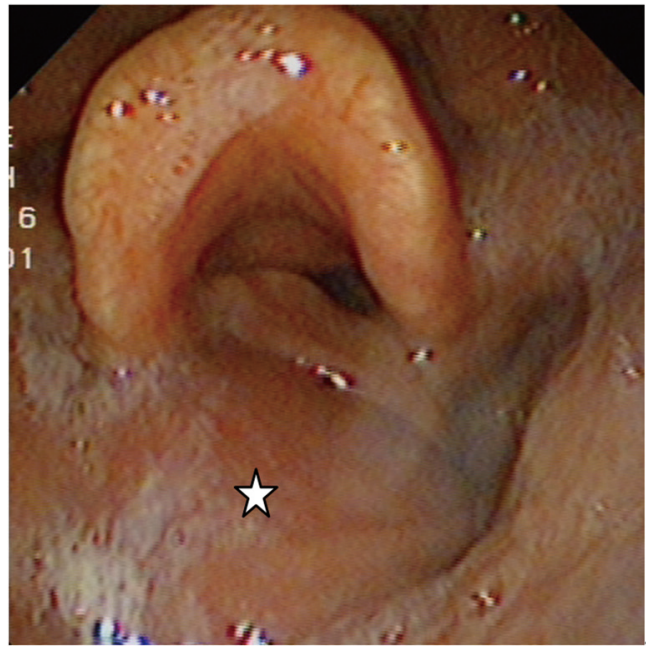

Figure 1 Preoperative fibrolaryngoscopy revealed a bulge (star)in the left lateral and posterior hypopharyngeal wall.

of recognizing the neurovascular anomalies in transoral and open surgery in the head and neck area. This case is presented in accordance with the CARE reporting checklist (available at http://dx.doi.org/10.21037/gs-20-663).

\section{Case presentation}

A 64-year-old Chinese woman was referred to our hospital with obstructive swallowing sensation and hoarseness for half a year. The patient had a past history of hypertension with good control but had not undergone surgery. Physical examination revealed a solid nodule in the left thyroid gland. Fibrolaryngoscopy revealed a bulge in the left lateral and posterior hypopharyngeal wall and vocal cord fixation of the same side (Figure 1). Contrast-enhanced computed tomography (CT) scan of the neck showed a lesion of $7 \mathrm{~cm}$ in length with heterogeneous enhancement in the left thyroid lobe invading the hypopharynx and paraglottic space, an aberrant right subclavian artery arising from the aortic arch, and aberrant right extracranial ICA with minimal distance $(1 \mathrm{~mm})$ to the pharyngeal wall (Figure 2). Fine needle aspiration biopsy from the left thyroid nodule confirmed papillary thyroid carcinoma (PTC).

The patient underwent total thyroidectomy, partial hypopharyngectomy, neck dissection (level II, III, IV of left side, and VI), and tracheotomy. The left RLN was sacrificed because it was invaded by the tumor. Meticulous dissection of the right thyroid lobe was performed because of aberrant neurovascular anatomy indicated on the preoperative CT imaging. We also identified a right NRLN originating from the vagus nerve above the level of the cricothyroid junction and entering the larynx (Figure 3). The defect of the hypopharynx was repaired with primary closure. The tumor size was approximately $6.5 \mathrm{~cm} \times 3.5 \mathrm{~cm} \times 3 \mathrm{~cm}$, and many calcifications were visible (Figure 4). The histopathological report showed thyroid papillary carcinoma of the left lobe, invading surrounding fat, skeleton muscle, mucosa of the hypopharynx, and positive lymph nodes in levels III, IV, and VI of the left side. The right thyroid gland showed a nodular goiter. The tumor stage of this patient was thyroid papillary carcinoma T4aN1bM0.

The patient did not present severe complications postoperatively. Hypocalcemia was treated by intravenous supplements (calcium gluconate $2 \mathrm{~g}$ per day) for one week and oral vitamin D and calcium (Caltrate $600 \mathrm{mg}$ Bid) for 1 month. Radioactive iodine therapy was applied after the surgery. At the 3-year follow-up, she remained cancerfree, and the hoarseness has improved. Fibrolaryngoscopy showed smooth mucosa of the hypopharynx and fixed left vocal cord (Figure 5). The timeline of the whole process of this patient is outlined in Figure 6.

All procedures performed in studies involving human participants were in accordance with the ethical standards of the institutional and/or national research committee(s) and with the Helsinki Declaration (as revised in 2013). Written informed consent was obtained from the patient.

\section{Discussion}

PTC comprises $80 \%$ of all patients with thyroid cancer, and patients with well-differentiated PTC have an excellent 5 -year survival rate of $>97 \%$ (4). However, dealing with advanced PTC invading the surrounding upper aerodigestive tract, such as trachea, esophagus, and rarely larynx and hypopharynx is challenging. At the first visit of the patient, we considered that the mass originated from neurogenic lesions under mucosa or minor salivary gland instead of thyroid as examined by fiberoptic laryngoscopy. The CT scan images indicated that the neoplasm arising from the thyroid gland invaded the left hypopharynx through posterior thyroid cartilage and narrowed paraglottic space. This mode of invasion was infrequent, and the first case in our department, which was the reason for neglecting the thyroid disease during the first visit. This case emphasized the necessity of the CT scan as the preoperative examination for some cases of PTC.

A right NRLN was confirmed during the surgery based 

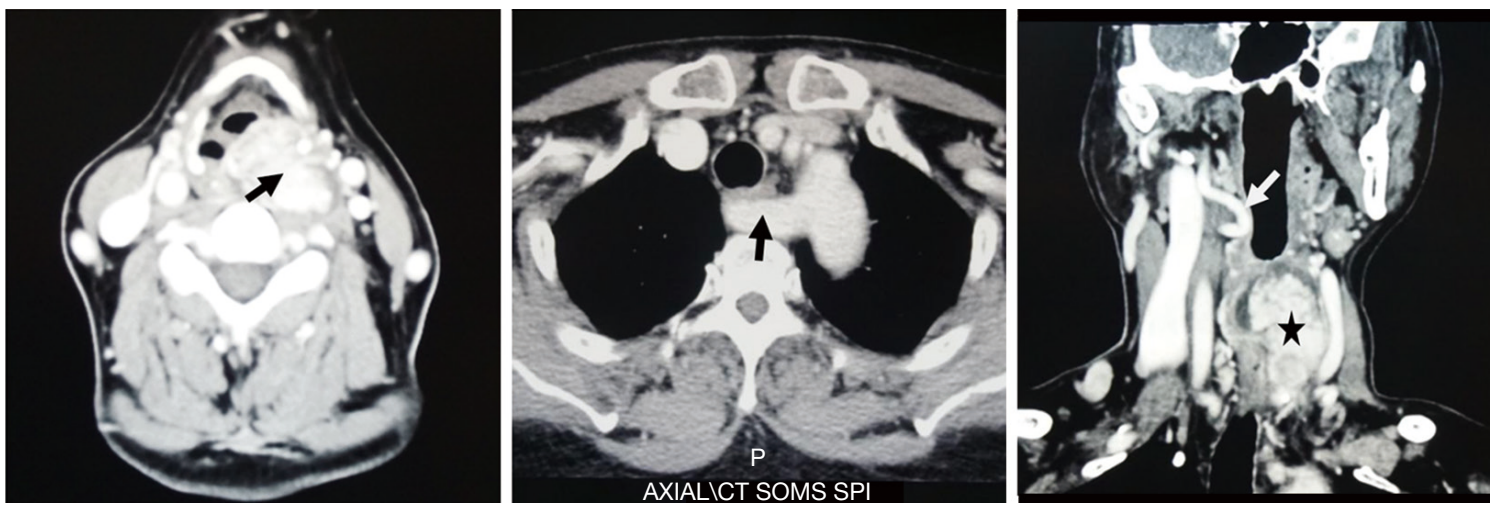

Figure 2 Enhanced CT scan. (A) The mass (arrow) from the left lobe of the thyroid gland invaded the hypopharynx with thick calcification inside. (B) The right subclavian artery originated from the aortic arch and ran between the vertebra and the esophagus (arrow). (C) An aberrant right extracranial internal carotid artery (arrow) with minimal distance $(1 \mathrm{~mm})$ to the pharyngeal wall. The length of the tumor was about $7 \mathrm{~cm}$ (star).

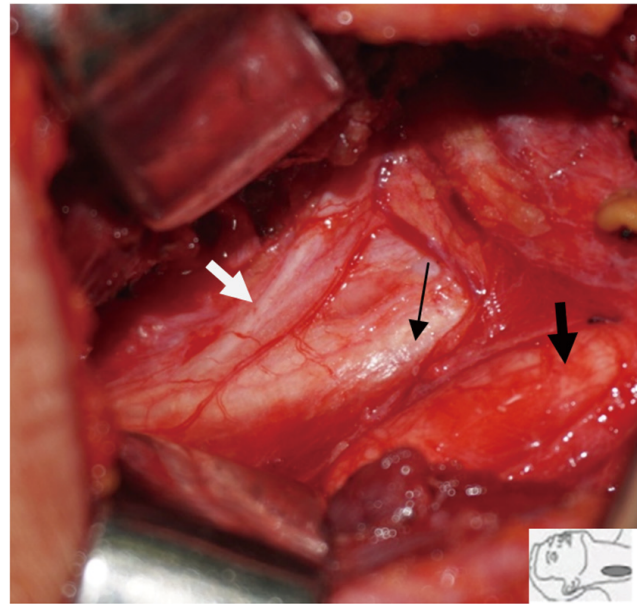

Figure 3 NRLN of the right side was found during the operation (the black solid arrow: right common carotid artery, the black arrow: vagus nerve, the white solid arrow: right NRLN). NRLN, non-recurrent laryngeal nerve.

on our preoperative discussion of the CT scan. The RLN originates from the sixth branchial arch and is related to the sixth arch artery. During embryonic development, the fifth and sixth arch arteries on the right side regress, and the fourth arch artery becomes the initial part of the right subclavian artery. On the left side, the sixth arch artery remains the arterial ligament, and the fourth arch artery evolves as a part of the aortic arch. Therefore, the RLNs arise from the vagus nerve in the mediastinum and return to the neck around the aortic arch in the left side and around the subclavian artery on the right side. In the

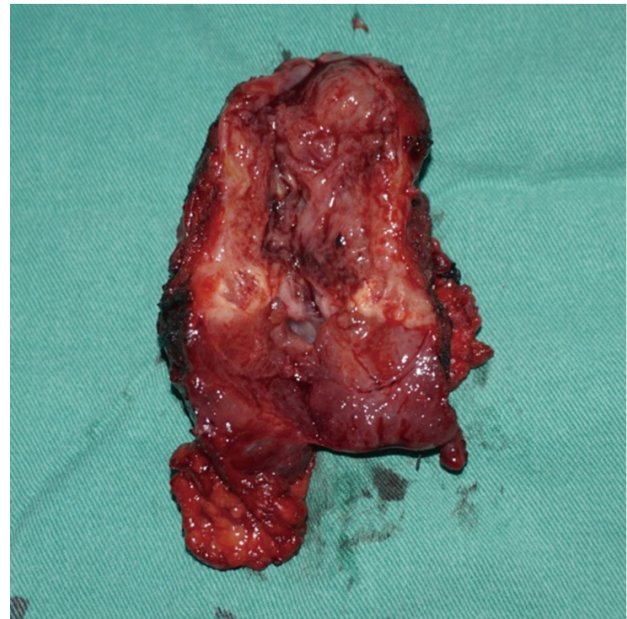

Figure 4 View of specimen. The tumor size was approximately $6.5 \mathrm{~cm} \times 3.5 \mathrm{~cm} \times 3 \mathrm{~cm}$, and many calcifications were visible.

case of regression of the fourth arch artery in the right side, the right NRLN occurs, while the right subclavian artery originates from the left side of the aortic arch and runs an abnormal course between the esophagus and the vertebra (3). The NRLN on the left side accounts for only $0.04 \%$ (2), which is extremely uncommon and associated with regression of the left fourth arch artery; this leads to the right aortic arch and is accompanied by the simultaneous occurrence of situs inversus.

According to the embryological basis, an aberrant right subclavian artery is coexistent with right NRLN. This case verified that the specific vascular structures of 


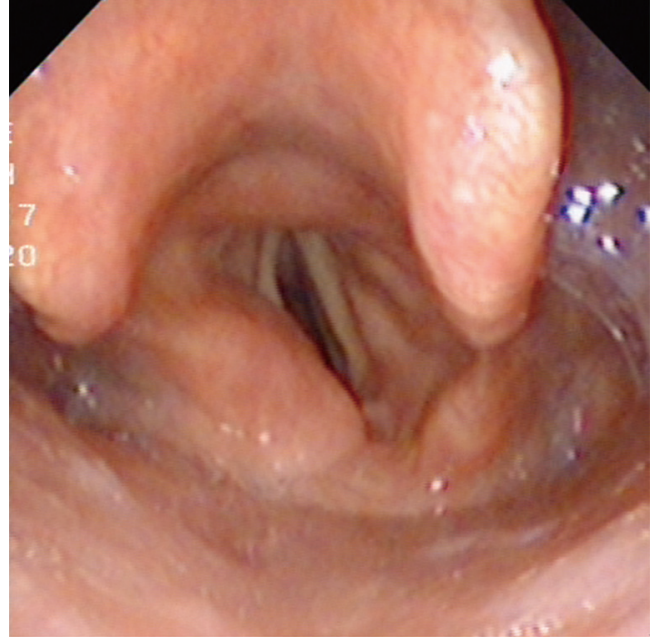

Figure 5 View of laryngoscopy of 3-year follow-up: smooth mucosa of hypopharynx and fixed left vocal cord.

CT findings were useful for the preoperative prediction of NRLN, as reported previously (5). NRLN could be detected by three methods: CT scan, ultrasonography, and intraoperative nerve monitoring (IONM) (5-7). CT scan and ultrasonography are based on the absence of the brachiocephalic trunk and presence of an aberrant subclavian artery, as described previously. A contrastenhanced CT scan is not recommended as the initial imaging for most patients with thyroid nodules, making preoperative ultrasonography a simple, non-invasive, and effective method to detect NRLN. In the ultrasonographic view of normal arterial anatomy, the bifurcation of the brachiocephalic trunk into the right subclavian artery and the right common carotid artery describes a Y. The absence of the Y sign predicted an NRLN with a sensitivity and specificity varying between $99 \%$ and $100 \%$ and $41 \%$ and $100 \%$, respectively (6). Despite these tests, the detection of NRLN prior to surgery may be difficult, especially when there is limited preoperative imaging to indicate the associated vascular anomaly. Currently, IONM is widely used during thyroid and parathyroid surgery, and it also facilitates intraoperative NRLN management (7). Since NRLN arises from the vagus nerve between the superior border of the thyroid cartilage superiorly and the fourth tracheal ring inferiorly, the presence of positive electromyographic response to the proximal stimulation of the superior border and the absence of response to the distal stimulation of the inferior border identifies NRLN (7). In order to avoid permanent vocal cord paralysis, the prediction of NRLN prior to surgical dissection is crucial, especially in patients similar to our case, wherein the other side of RLN is sacrificed because of tumor invasion.

We also found a right aberrant extracranial ICA according to the images of the CT scan. An aberrant extracranial ICA is not a rare anatomic variation and can be detected in about $5-6 \%$ of the general population (8). The occurrence of this malformation is also associated with abnormal embryological development of the arch arteries or age-related degenerative changes in the vessel wall. During fetal development, a loop is formed between the third arch artery and the dorsal aorta during week 5 of development. When the heart descents into the mediastinum, the loop is straightened, and hence, the variations in this process may result in an aberrant ICA (1). In this patient, we suspect the embryological reason because of the concurrence of an aberrant right subclavian artery, which is also associated with the anomaly of the arch arteries. An aberrant extracranial ICA is classified as a benign anomaly that does not need treatment unless the patient has signs of cerebrovascular ischemia (1). Weibel and Fields classified this variation into three types: tortuosity, kinking, and coiling (9). Kinking is characterized by a sharp angulation of the two segments of extracranial ICA $<90^{\circ}$, which is fulfilled by this represented case.

It is imperative for clinicians to recognize aberrant extracranial ICAs to prevent severe complications during laryngoscopy, biopsy, and surgical excision. Several studies highlighted that ICA constitutes a lethal complication as a result of hemorrhage from aberrant ICAs during pharyngeal surgeries if the artery is associated with the pharyngeal wall $(1,8)$. Under other circumstances, the artery could shift laterally, and there is a risk of vascular injury during open neck surgery, especially neck dissection. In our center, one patient with PTC suffered from severe cerebrovascular complications during lateral neck dissection because the surgeon did not realize the tortuous ICA had exceeded the lateral wall of the internal jugular vein and resulted in unexpected injury. Hence, the patients should be evaluated meticulously in order to prevent life-threatening complications if physicians plan an intervention to the neck or pharynx. Therefore, a new clinicoradiological classification system was proposed according to two critical parameters: the vessel's minimal distance to the pharyngeal wall and the corresponding pharyngeal level (8). In the current case, neck dissection was not planed in the right side, and hence, the right aberrant extracranial ICA was not a concern. 


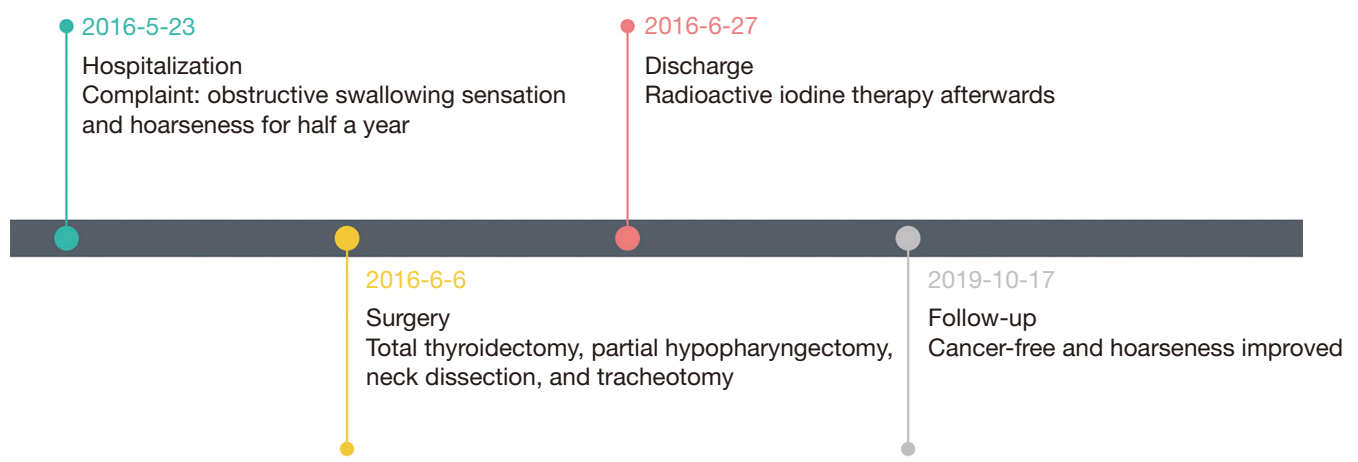

Figure 6 The timeline of the medical history of this patient.

There are some pitfalls in this case. The endoscope appearance makes it prone to be misdiagnosed as hypopharyngeal neoplasm. Because of tumor invasion of the left RLN, it is crucial to preserve the NRLN in the right side, which is associated with a high risk of nerve injury if preoperative prediction is not achieved. Meanwhile, the right aberrant extracranial ICA was noticed in the CT scan with minimal distance $(1 \mathrm{~mm})$ to the pharyngeal wall, which physicians should be aware of while performing transoral procedure if necessary.

In conclusion, it is uncommon that advanced PTC invades the hypopharynx through posterior thyroid cartilage. Additionally, this is the first case report of concurrent aberrant extracranial ICA and NRLN. These two variants may occur simultaneously because of similar etiology of the embryological maldevelopment of arch arteries. Thus, a careful physical, endoscopic, and radiological examination to recognize these anatomical variants are essential for clinicians to prevent severe or lethal complications during surgery.

\section{Acknowledgments}

Funding: None.

\section{Footnote}

Reporting Checklist: The authors have completed the CARE reporting checklist. Available at http://dx.doi.org/10.21037/ gs-20-663

Conflicts of Interest: All authors have completed the ICMJE uniform disclosure form (available at http://dx.doi. org/10.21037/gs-20-663). The authors have no conflicts of interest to declare.
Ethical Statement: The authors are accountable for all aspects of the work in ensuring that questions related to the accuracy or integrity of any part of the work are appropriately investigated and resolved. All procedures performed in studies involving human participants were in accordance with the ethical standards of the institutional and/or national research committee(s) and with the Helsinki Declaration (as revised in 2013). Written informed consent was obtained from the patient. Our institution's Research Ethics Board does not require a review or approval for case reports.

Open Access Statement: This is an Open Access article distributed in accordance with the Creative Commons Attribution-NonCommercial-NoDerivs 4.0 International License (CC BY-NC-ND 4.0), which permits the noncommercial replication and distribution of the article with the strict proviso that no changes or edits are made and the original work is properly cited (including links to both the formal publication through the relevant DOI and the license). See: https://creativecommons.org/licenses/by-nc-nd/4.0/.

\section{References}

1. Paulsen F, Tillmann B, Christofides C, et al. Curving and looping of the internal carotid artery in relation to the pharynx: frequency, embryology and clinical implications. J Anat 2000;197 Pt 3:373-81.

2. Henry BM, Sanna S, Graves MJ, et al. The NonRecurrent Laryngeal Nerve: a meta-analysis and clinical considerations. PeerJ 2017;5:e3012.

3. Henry JF, Audiffret J, Denizot A, et al. The nonrecurrent inferior laryngeal nerve: review of 33 cases, including two on the left side. Surgery 1988;104:977-84.

4. Schneider DF, Chen H. New developments in the 
diagnosis and treatment of thyroid cancer. CA Cancer J Clin 2013;63:374-94.

5. Lee YS, Son EJ, Chang HS, et al. Computed tomography is useful for preoperative identification of nonrecurrent laryngeal nerve in thyroid cancer patients. Otolaryngol Head Neck Surg 2011;145:204-7.

6. Citton M, Viel G, Iacobone M. Neck ultrasonography for detection of non-recurrent laryngeal nerve. Gland Surg 2016;5:583-90.

7. Kamani D, Potenza AS, Cernea CR, et al. The

Cite this article as: Wen F, Xie R, Shen H, Zhao E. A case report of concurrence of aberrant extracranial internal carotid artery and nonrecurrent laryngeal nerve in a patient of advanced thyroid carcinoma invading hypopharynx. Gland Surg 2021;10(5):1786-1791. doi: 10.21037/gs-20-663 nonrecurrent laryngeal nerve: anatomic and electrophysiologic algorithm for reliable identification. Laryngoscope 2015;125:503-8.

8. Pfeiffer J, Becker C, Ridder GJ. Aberrant extracranial internal carotid arteries: New insights, implications, and demand for a clinical grading system. Head Neck 2016;38 Suppl 1:E687-93.

9. Weibel J, Fields WS. Tortuosity, coiling, and kinking of the internal carotid artery. I. Etiology and radiographic anatomy. Neurology 1965;15:7-18. 